\title{
Erratum to: Eosinophilic coronary periarteritis (vasospastic angina and sudden death), a new type of coronary arteritis: report of seven autopsy cases and a review of the literature
}

\author{
Hiroki Kajihara • Yoshiro Tachiyama • \\ Takanori Hirose • Aya Takada • Kazuyuki Saito • \\ Tatsuya Murai • Wataru Yasui
}

Published online: 14 February 2013

(C) Springer-Verlag Berlin Heidelberg 2013

Erratum to: Virchows Arch

\section{DOI 10.1007/s00428-012-1351-7}

The original version of this article, unfortunately, contained an error.

Aya Takada's surname was incorrectly spelled as Takata. The correct spelling is presented in this paper.

The online version of the original article can be found at http://dx.doi.org/ 10.1007/s00428-012-1351-7.

H. Kajihara $(\bowtie)$

Division of Tumor Registry, Hiroshima Prefectural Medical

Association, Kannon-honmachi1-1-1,

Nishi-Ku, Hiroshima 733-8540, Japan

e-mail: kajihara@sky.megaegg.ne.jp

Y. Tachiyama

Division of Pathology, NHO Hiroshima Nishi Medical Center,

Kuba 4-1-1,

Otake City, Hiroshima 739-0696, Japan

T. Hirose

Division of Pathology, Tokushima Prefectural Central Hospital,

Kuramotocho 1-10-3,

Tokushima 770-8539, Japan

A. Takada $\cdot$ K. Saito

Department of Forensic Medicine, Saitama Medical School, Morohongo 38, Moroyamacho,

Irima-gun, Saitama 350-0495, Japan

T. Murai

Division of Pathology, Sakakibara Heart Institute,

Asahicho 3-16-1,

Fuchu City, Tokyo 183-0003, Japan

W. Yasui

Department of Molecular Pathology, Hiroshima University Institute of Biomedical and Health Sciences, Kasumi 1-2-3,

Minami-Ku, Hiroshima 734-8551, Japan 